PROCEEDINGS OF THE

AMERICAN MATHEMATICAL SOCIETY

Volume 133, Number 9, Pages 2805-2812

S 0002-9939(05)07818-4

Article electronically published on March 22, 2005

\title{
AN APPROACH TO THE REGULARITY FOR STABLE-STATIONARY HARMONIC MAPS
}

\author{
HSU DELIANG
}

(Communicated by Richard A. Wentworth)

\begin{abstract}
In this paper we investigate the regularity of stable-stationary harmonic maps. By assuming that the target manifolds do not carry any stable harmonic $S^{2}$, we obtain some compactness results and regularity theorems. In particular, we prove that the Hausdorff dimension of the singular set of these maps cannot exceed $n-3$, and the dimension estimate is optimal.
\end{abstract}

\section{INTRODUCTION}

In this paper we are centered on the regularity of stationary harmonic maps. Many important contributions to the regularity theory of minimizing harmonic maps have been made. It has been established by Schoen-Uhlenbeck [15] for general cases, and independently by Giaquinta-Giusti [4] for the case where the image of maps is contained in a single chart, that any minimizing harmonic map in $H^{1,2}(M, N)$ is smooth except for a singular set where the Hausdorff dimension of the singular set is less than or equal to $n-3$. Recently, inspired by Hélein's beautiful work [6] on weakly harmonic maps from a two-dimensional domain, it was proved by Evans [3] in the case with values into spheres and by Bethuel [2] in general that any stationary harmonic map is smooth away from a singular set $Z$ with $\mathcal{H}^{n-2}(Z)=0$ where $\mathcal{H}^{n-2}$ denotes $(n-2)$-dimensional Hausdorff measure. Another major work in this direction was done by Fanghua Lin [10; by a technical analysis of the singular set, he found the stratification phenomenon and proved that if the target manifold does not carry any harmonic $S^{2}$, then the singular sets of stationary harmonic maps are $m \leq n-4$ rectifiable. On the other hand, Rivière [13] constructed a weakly harmonic map which has singularities almost everywhere in $M$, but it is not stationary. All known examples of stationary weak harmonic maps have singular set $Z$ with $\operatorname{dim}(Z) \leq n-3$. An interesting open problem is whether one can improve the estimate of the Hausdorff dimension of the singular set for stationary harmonic maps; it was conjectured by R.Hardt, see the survey 11], that the singular sets have the same Hausdorff dimension estimate as in the minimizing harmonic map' case. Recently, Hong [7] and Hong and Wang [8 gave a partial answer to this problem. They get the optimal estimate of stationary harmonic maps from $M$ into $S^{k}(k \geq 4)$ under the 'stable' condition or in the stable

Received by the editors December 15, 2003 and, in revised form, May 4, 2004.

2000 Mathematics Subject Classification. Primary 58E20.

Key words and phrases. Stable harmonic map, stationary harmonic map, blowing-up map.

The author was supported in part by Chinese NSF Grant 10301020. 
inequality condition. In this paper, we give a different approach to regularity of stationary harmonic maps. In contrast to the work of Hong and Wang [8], we use the stratification method and get a more geometrical condition to ensure compactness of the sequence of stationary harmonic maps, and finally get the optimal estimate of the Hausdorff dimension of the singular set. Our methods here are based on Lin's recent work [10]. Furthermore, in another paper [5] we get a similar result for the regularity of $k$-indexed stationary harmonic maps.

We assume that $n, m$ are integers with $n \geq 3, \Omega$ is a domain of $\mathbf{R}^{n}$ with smooth boundary, and $N$ denotes an $m$-dimensional compact Riemannian manifold which may be considered as embedded in $\mathbf{R}^{l}$ by Nash's theorem. We define the space $H^{1,2}(\Omega, N)$ by

$$
H^{1,2}(\Omega, N)=\left\{u \mid u \in H^{1,2}\left(\Omega, \mathbf{R}^{l}\right), u(x) \in N \text {, for a.e. } x \in \Omega\right\}
$$

and define the Dirichlet energy of $u \in H^{1,2}(\Omega, N)$ by $E(u, \Omega)=\frac{1}{2} \int_{\Omega}|\nabla u|^{2} d x$, where $\nabla u$ is the gradient of $u$.

We recall that $u: \Omega \rightarrow N$ is a weakly harmonic map if $u$ belongs to $H^{1,2}(\Omega, N)$ and satisfies

$$
-\Delta u=A(\nabla u, \nabla u)
$$

in the sense of distribution, where $A(\cdot, \cdot)$ denotes the second fundamental form of $N$ embedded in $\mathbf{R}^{l}$.

We say that a harmonic map $u$ is stable if the second variation of $E(u, \Omega)$ is nonnegative, i.e.

$$
\left.\frac{d^{2}}{d t^{2}}\right|_{t=0} E\left(\Phi_{t}\right)=I(V, V)=\int_{\Omega}\left[|\nabla V|^{2}+\left\langle\left(R^{N} \cdot u\right)\left(V, d u_{-}\right) V, d u\right\rangle\right] d y \geq 0
$$

for any $V \in \Gamma\left(u^{-1}(T N)\right)$ with compact support, where $\Phi_{t}: \Omega \longmapsto N$ with $\Phi_{0}=u$, $V=\left.\frac{\partial}{\partial t}\right|_{t=0} \Phi_{t}$, and

$$
\left\langle R^{N} \cdot \Phi\left(V, d u_{-}\right) V, d u\right\rangle=-\sum_{i} g_{u(y)}^{N}\left(R_{u(y)}\left(\partial_{i} u(y), V(y)\right) V(y), \partial_{i} u(y)\right) .
$$

The index of a harmonic map $u$ (denoted by $\operatorname{Ind}(u)$ ) is the maximal dimension of a linear subspace of $\Gamma\left(u^{-1}(T N)\right)$ on which the second variation form of $E(u, \Omega)$ is negative definite. The nullity of $u$ is the dimension of the subspace of Jacobi fields, an element $U \in \Gamma\left(u^{-1}(T N)\right)$ being called a Jacobi field if

$$
I(U, V)=0, \quad \text { for all } V \in \Gamma\left(u^{-1}(T N)\right) .
$$

We say that a harmonic map $u$ is stationary if for each vector field $\eta \in C^{1}\left(\Omega, \mathbf{R}^{n}\right)$ having compact support in $\Omega$, setting $u^{t}=u(x+t \eta(x))$, we have

or in other words,

$$
\left.\frac{d}{d t}\right|_{t=0} E\left(u^{t}, \Omega\right)=0
$$

$$
\int_{\Omega} \sum_{i, j=1}^{n}\left[\delta_{i, j}|\nabla u|^{2}-2 D_{i} u D_{j} u\right] D_{i} \eta^{j} d x=0 .
$$

We give the definition of a stable-stationary weakly harmonic map.

Definition 1.1. A function $u \in H^{1,2}(\Omega, N)$ is a stable-stationary weakly harmonic map if $u$ is a weakly harmonic map, stationary and stable. 
Our main result is:

Theorem 1.2. Let $u \in H^{1,2}(\Omega, N)$ be a stable-stationary weakly harmonic map. Then if the target manifold $N$ does not carry any stable harmonic map from $S^{2}$ to $N$, the Hausdorff dimension of the singular set of $u$ is less than or equal to $n-3$.

\section{A COMPACTNESS LEMma AND PROOF OF THE THEOREM}

In this section, we give the proof of Theorem 2 . We need the following result for stationary harmonic maps, which can be found in the work of Lin [10].

Lemma 2.1. Let $u_{i} \in H^{1,2}(\Omega, N)$ be a sequence of stationary harmonic maps, with $\int_{\Omega}\left|\nabla u_{i}\right|^{2}$ uniformly bounded. Denote

$$
\Sigma=\bigcap_{r>0}\left\{x \in \Omega: \liminf _{i \rightarrow \infty} r^{2-m} \int_{B_{r}(x)}\left|\nabla u_{i}\right|^{2} \geq \varepsilon_{0}^{2}\right\},
$$

where $\varepsilon_{0}$ is the constant in the small energy regularity (15], Theorem 2.2; see also 2], Theorem I.4). Then if $\mathcal{H}^{n-2}(\Sigma)>0$, there exists a nonconstant, smooth harmonic map from $S^{2}$ into $N$.

We call this map the blowing-up harmonic map and denote it by $u_{b}$. Now, we are ready to prove the following key lemma.

Lemma 2.2. Let $u_{i} \in H^{1,2}(\Omega, N)$ be stable-stationary harmonic maps, with $\int_{\Omega}\left|\nabla u_{i}\right|^{2}$ uniformly bounded. Then if the target manifold does not carry any stable harmonic map from $S^{2}$ into $N$, there exists a subsequence of $\left\{u_{i}\right\}$ converging strongly to a stable-stationary harmonic map in $H_{l o c}^{1,2}(\Omega, N)$.

The similar compactness lemma for a stable-stationary harmonic map sequence was obtained in Hong and Wang [8] by assuming stable inequality. To prove Lemma 2.2 , we need to prove the following lemma.

Lemma 2.3. Let $u_{i} \in H^{1,2}(\Omega, N)$ be a sequence of stationary harmonic maps, with $\int_{\Omega}\left|\nabla u_{i}\right|^{2}$ uniformly bounded. Then if the $u_{i}$ are stable harmonic maps for each $i$ and $\mathcal{H}^{n-2}(\Sigma)>0$, then the blowing-up harmonic map $u_{b}$ from $S^{2}$ into $N$ must be stable.

Proof. We can always assume that $u_{i} \rightarrow u_{0}$ weakly in $H^{1,2}(\Omega, N)$ and that $\mu_{i}=$ $\left|\nabla u_{i}\right|^{2} \rightarrow \mu=\left|\nabla u_{0}\right|^{2}+\nu$ in the sense of measure as $i \rightarrow \infty$. Because $\mathcal{H}^{n-2}(\Sigma)>0$, the dimension reduction and Lin's theorem ([10], Theorem $\mathrm{C}$ ) imply that $\Sigma$ is a closed $\mathcal{H}^{n-2}$-rectifiable set. Then by a lemma in Lin's work ([10], Lemma 2.1) we conclude that there exist $r_{k}, x_{k}$ with $r_{k} \rightarrow 0$ as $k \rightarrow \infty$ and $x_{k} \rightarrow x_{0} \in \Sigma$, such that $\mu_{r_{k}, x_{k}} \rightarrow \mu_{*}$ in the sense of measure. Here the Radon measure $\mu_{*}=C_{0} \mathcal{H}^{n-2}\left\lfloor\Sigma_{*}\right.$ and $\Sigma_{*}=\mathbf{R}^{n-2} \times\{0\}, \mu_{r_{k}, x_{k}}(A)=\mu\left(x_{k}+r_{k} A\right) r_{k}^{n-2}$. Denoting $u_{i}^{k}(x)=u_{i}\left(x_{k}+r_{k} x\right)$, by the diagonal subsequence argument, we can get a subsequence of $u_{i}^{k}(x)$ which we denote by $u_{i}^{i}(x)$ such that $\left|\nabla u_{i}^{i}(x)\right|^{2} \rightarrow \mu_{*}=C_{0} \mathcal{H}^{n-2}\left\lfloor\Sigma_{*}\right.$ in the sense of measure. Because $u_{i}(x)$ are stationary harmonic maps, so $u_{i}^{i}(x)$ are also stationary harmonic maps; and if $u_{i}(x)$ are stable harmonic maps, then $u_{i}^{i}(x)$ are also stable. Set

$$
F_{i}(x)=\int_{B_{2}^{1} \times\{0\}} \sum_{k=1}^{n-2}\left|\frac{\partial u_{i}^{i}}{\partial x_{k}}\right|^{2}\left(x, x^{\prime}\right) d x^{\prime}
$$


for $x \in \Sigma_{*} \cap B_{2}^{n}(0)=B_{2}^{2}(0) \times\{0\}$. Here and in what follows we denote by $B_{r}^{n}(y)$ the metric ball centered at $y$ with radius $r$ in $\mathbf{R}^{n}, B_{r}^{1}(y)$ the metric ball centered at $y$ with radius $r$ in $\mathbf{R}^{n-2}$ and $B_{r}^{2}(y)$ the metric ball centered at $y$ with radius $r$ in $\mathbf{R}^{2}$. Then as in the proof of Lemma 2.1 in Lin's work ([10, Lemma 3 ) or by Lemma 2.2 ([9], Lemma 2.2), we get

$$
F_{i}(x) \rightarrow 0, \quad \text { as } i \rightarrow \infty .
$$

We consider the Hardy-Littlewood function $M_{F_{i}}(x)$ of $F_{i}(x)$, which is defined by

$$
M_{F_{i}}(x)=\sup _{0<r<1} r^{2-n} \int_{B_{r}^{1}(x)} F_{i}(x) d x .
$$

By the weak type $(1,1)$ inequality for $M_{F_{i}}(x)$, we have

$$
\mathcal{H}^{n-2}\left\{x \in B_{2}^{1}(0) \mid M_{F_{i}}(x) \geq \lambda\right\} \leq \frac{C}{\lambda} \int_{B_{2}^{1}(0) \times B_{2}^{2}(0)} \sum_{k=1}^{n-2}\left|\frac{\partial u_{i}^{i}}{\partial x_{k}}\right|^{2} d x d x^{\prime}
$$

for any $\lambda>0$. So from (2.2) we have

$$
\lim _{i \rightarrow \infty} \mathcal{H}^{n-2}\left\{x \in B_{2}^{1}(0) \mid M_{F_{i}}(x) \geq \lambda\right\}=0 .
$$

As in the proof of Lemma 3.1 of Lin's work ([10], Lemma 3.1), by the partial regularity theorem of Bethuel [2] for stationary harmonic maps and (2.3), we can find a sequence of points $\left\{x_{i}^{1}\right\}, i=1,2, \cdots, x_{i}^{1} \in B_{1}^{1}(0)$, such that

$$
u_{i}^{i}\left(x, x^{\prime}\right) \text { is smooth near all }\left(x_{i}^{1}, x^{\prime}\right) \in B_{1}^{1}(0) \times B_{1}^{2}(0),
$$

and that

$$
\sup _{0<r \leq 1} r^{2-n} \int_{B_{r}^{1}\left(x_{i}^{1}\right)} F_{i}(x) d x \rightarrow 0, \quad \text { as } i \rightarrow \infty .
$$

Also for all $i$ sufficiently large, we may find $x_{i}^{\prime}$ and $\delta_{i}$ with $x_{i}^{\prime} \in B_{\frac{1}{4}}^{2}(0)$ and $\delta_{i} \in\left(0, \frac{1}{2}\right)$, which satisfy that the maximum

$$
\max _{x^{\prime} \in B_{\frac{1}{2}}^{2}(0)} \delta_{i}^{2-n} \int_{B_{\delta_{i}}^{1}\left(x_{i}^{1}\right) \times B_{\delta_{i}}^{2}\left(x^{\prime}\right)}\left|\nabla u_{i}^{i}\right|^{2} d x d x^{\prime}=\frac{\varepsilon_{0}}{2^{n+3}}
$$

is achieved at $x_{i}^{\prime}$, and $\delta_{i} \rightarrow 0$, as $i \rightarrow \infty$ (for the details of how to get the existence of $x_{i}^{\prime}$ and $\delta_{i}$ one may see the proof of Lemma 3.1 in Lin's work [10]).

Set

$$
v_{i}(y)=u_{i}^{i}\left(p_{i}+\delta_{i} y\right)=u_{i}\left(q_{i}+\gamma_{i} x\right), \quad p_{i}=\left(x_{i}^{1}, x_{i}^{\prime}\right),
$$

for some $q_{i} \in \Omega$, and $\gamma_{i} \rightarrow 0$, as $i \rightarrow \infty$. From the assumption of the lemma we know that if $u_{i}(x)$ are stable harmonic maps, then $v_{i}(y)$ are also stable harmonic maps. That is,

$$
\int_{\Omega_{i}}\left[|\nabla V|^{2}+\left\langle\left(R^{N} \cdot u\right)\left(V, d v_{i-}\right) V, d v_{i}\right\rangle\right] d y \geq 0
$$

for any $V \in \Gamma\left(v_{i}^{-1}(T N)\right)$ with compact support (here $\left.\Omega_{i}=\frac{1}{\gamma_{i}}\left(\Omega-q_{i}\right)\right)$. Furthermore, the $v_{i}(y)$ are stationary harmonic maps defined on

$$
B_{R_{i}}^{1}(0) \times B_{R_{i}}^{2}(0) \supset B_{3}^{1}(0) \times B_{R_{i}}^{2}(0),
$$


where $R_{i}=\frac{1}{4 \delta_{i}} \rightarrow \infty$, as $i \rightarrow \infty$. From (2.5), (2.6) and energy monotonicity for both $v_{i}$ and $u_{i}^{i}$, we have the following properties of $v_{i}$ :

$$
\begin{aligned}
& \int_{B_{R_{i}}^{1}(0) \times B_{R_{i}}^{2}(0)} \sum_{k=1}^{n-2}\left|\frac{\partial v_{i}}{\partial y_{k}}\right|^{2} d y \rightarrow 0 \quad \text { as } i \rightarrow \infty, \\
& \frac{\varepsilon_{0}}{2^{n+3}}=\int_{B_{1}^{1}(0) \times B_{1}^{2}(0)}\left|\nabla v_{i}\right|^{2} d y \\
& =\max _{x^{\prime} \in B_{R_{i}-1}^{2}}\left\{\int_{B_{1}^{1}(0) \times B_{1}^{2}\left(x^{\prime}\right)}\left|\nabla v_{i}\right|^{2} d y\right\}, \\
& \sup _{i}\left\{\int_{B_{R}^{1}(0) \times B_{R}^{2}(0)}\left|\nabla v_{i}\right|^{2} d y\right\} \leq C R^{n-2}
\end{aligned}
$$

for $0<R<R_{i}$. Moreover, by Allard's Strong Constancy Lemma (1], pp. 3-5) as in Lin's work [10], one may get

$$
\int_{B_{2}^{1}(0) \times B_{2}^{2}(0)}\left|\nabla v_{i}\right|^{2}\left(x^{1}, x^{\prime}+b\right) d x^{1} d x^{\prime} \leq \varepsilon_{0},
$$

for all $b \in B_{R_{i}-2}^{2}(0)$. Therefore by the small energy regularity theorem [2], we have $v_{i} \rightarrow u_{b}$ (we also denote by $v_{i}$, one may take a subsequence if necessary) in

$$
C^{1, \alpha}\left(B_{\frac{3}{2}}^{1}(0) \times B_{R_{i}-1}^{2}(0)\right) \quad \text { as } i \rightarrow \infty .
$$

The limiting map $u_{b}$ is a smooth harmonic map defined on $B_{\frac{3}{2}}^{1}(0) \times \mathbf{R}^{2}$ such that

$$
\begin{gathered}
\int_{B_{1}^{1}(0) \times B_{1}^{2}(0)}\left|\nabla u_{b}\right|^{2} d x^{1} d x^{\prime}=\frac{\varepsilon_{0}}{2^{n+3}}, \\
\int_{\mathbf{R}^{n-2} \times \mathbf{R}^{2}} \sum_{k=1}^{n-2}\left|\frac{\partial u_{b}}{\partial y_{k}}\right|^{2} d y=0,
\end{gathered}
$$

and

$$
\int_{B_{R}^{1}(0) \times B_{R}^{2}(0)}\left|\nabla u_{b}\right|^{2} d y \leq C R^{n-2} .
$$

Moreover from (2.12) and (2.13), by the strong convergence of $v_{i} \rightarrow v_{b}$, we have

$$
\int_{\mathbf{R}^{2}}\left[|\nabla V|^{2}+\left\langle\left(R^{N} \cdot u\right)\left(V, d u_{b-}\right) V, d u_{b}\right\rangle\right] d y \geq 0,
$$

for any $V \in \Gamma\left(u_{b}^{-1}(T N)\right)$ with compact support. Therefore we get a smooth, nonconstant harmonic map $u_{b}: \mathbf{R}^{2} \rightarrow N$ of finite energy which satisfies (2.14). Hence by Sacks-Uhlenbeck's [14 theorem, $u_{b}$ is a smooth nonconstant harmonic map from $S^{2}$ to $N$ which is stable. This completes the proof of the lemma.

Lemma 2.2 is obvious from Lemma 2.3 .

Remark 2.4. Along the same line as the proof of Lemma 2.3, we can prove the following result. 
If $u_{i} \in H^{1,2}(\Omega, N)$ is a sequence of stationary harmonic maps, with a uniformly bounded energy satisfying

$$
\int_{\Omega}|\nabla \varphi|^{2} \geq A \int_{\Omega}\left|\nabla u_{i}\right|^{2}|\varphi|^{2}, \quad \forall \varphi \in C_{0}^{1}(\Omega, \mathbf{R}), \forall i,
$$

for some positive constant $A$, and $\mathcal{H}^{n-2}(\Sigma)>0$, then the blowing-up harmonic map $u_{b}$ also satisfies the stable inequality

$$
\int_{R^{2}}|\nabla \varphi|^{2} \geq A \int_{R^{2}}\left|\nabla u_{b}\right|^{2}|\varphi|^{2}, \quad \forall \varphi \in C_{0}^{1}\left(S^{2}, \mathbf{R}\right) .
$$

The following Liouville-type result governs the occurrence of blowing-up harmonic maps, which also illustrates some connection between the stable condition and stable inequality.

Proposition 2.5. If a harmonic map $u_{b}$ from $\mathbf{R}^{2}$ into $N$ satisfies the stable inequality (2.16), then $u_{b}$ must be constant.

Proof. We choose $\varphi_{t}\left(x^{\prime}\right)$ to be

$$
\varphi_{t}\left(x^{\prime}\right)=\left\{\begin{array}{cc}
1, & \text { on } B_{t}^{2}(0), \\
\frac{\ln \left(t^{2} / x^{\prime} \mid\right)}{\ln t}, & \text { on } B_{t^{2}}^{2}(0) \backslash B_{t}^{2}(0), \\
0, & \left|x^{\prime}\right|>t^{2} .
\end{array}\right.
$$

Substituting $\varphi_{t}\left(x^{\prime}\right)$ into (2.16) , we have

$$
\begin{aligned}
A \int_{\mathbf{B}_{t}(0)}\left|\nabla u_{b}\right|^{2}\left|\varphi_{t}\right|^{2} & \leq \int_{B_{t^{2}}^{2}(0) \backslash B_{t}^{2}(0)} \frac{1}{(\ln t)^{2}\left|x^{\prime}\right|^{2}} d x^{\prime} \\
& \leq C \frac{1}{(\ln t)} .
\end{aligned}
$$

Letting $t$ tends to $+\infty$, we conclude that $u_{b}$ must be constant.

For the further relation between the stable inequality and the stable condition one may see Wei's work 16.

Combining the above remark and proposition, we conclude that $H^{n-2}(\Sigma)=0$, and hence we give a different proof of the compactness lemma in Hong and Wang's work [8, which is a key step in their paper.

Proof of Theorem 2. Without loss of generality, assume that $u$ is a stable-stationary weakly harmonic map from $\Omega$ into $N$ where $N$ does not carry any stable harmonic maps $S^{2}$, and $Z$ is the singular set of $u$. Let $0 \leq s<n-2$ be such that $\mathcal{H}^{s}(Z)>0$. Then by the well-known density results, we have

$$
\lim _{r \rightarrow 0} \sup r^{-s} \mathcal{H}^{s}\left(Z \cap B_{r}^{n}(x)\right) \geq c>0
$$

for a sequence $r \rightarrow 0$. Assume the origin is one of the singular points and rescaling $u(x)$ as $u_{\lambda}(x)=u(\lambda x)$. By the well-known monotonicity inequality and Lemma 4 , there exists a subsequence $\lambda_{i}$ of $\lambda$ such that $u_{\lambda_{i}}(x)$ converges to $u_{0}$ strongly in $H^{1,2}\left(B_{\frac{1}{2}}, N\right)$ where $u_{0}$ is a tangent harmonic map, i.e., $\frac{\partial}{\partial r} u_{0}=0$ a.e. in $B_{1}$. Let 
$Z_{0}$ and $Z_{i}$ be the singular sets of $u_{0}(x)$ and $u_{\lambda_{i}}(x)$. By Lemma 5.3 of SchoenUhlenbeck [15] and (2.17), we obtain

$$
\mathcal{H}^{s}\left(Z_{0} \cap B_{\frac{1}{2}}\right) \geq \lim _{i \rightarrow \infty} \mathcal{H}^{s}\left(Z_{i} \cap B_{\frac{1}{2}}\right)=\lim _{i \rightarrow \infty} \lambda_{i}^{s} \mathcal{H}^{s}\left(Z \cap B_{\frac{\lambda_{i}}{2}}\right)>0 .
$$

Since $\frac{\partial u_{0}}{\partial r}=0$, we have $\lambda Z_{0} \subset Z_{0}$ for any $\lambda>0$. So we repeat the Federer reducing dimension argument (see Corollary 1.10 in [10]). As in [15], we find an integer $m$ and harmonic map $u_{j} \in H^{1,2}\left(\mathbf{R}^{n}, S^{k}\right)$ for $j=1, \cdots, m$ such that the $u_{j}$ are stable-stationary, $\frac{\partial u_{j}}{\partial x_{\alpha}}=0$ for $\alpha=1, \cdots, j$ and $\mathcal{H}^{s}\left(Z_{j} \cap B_{1}\right)>0$ where $Z_{j}$ is the singular set of $u_{j}$. We repeat the argument until $s-m \leq 0$. Since $s \leq n-2$, we then have $m \leq n-2$. It is impossible for $m=n-2$ since $\mathcal{H}^{n-2}(Z)=0$. Therefore we have $s \leq n-3$. This shows that the Hausdorff dimension of the singular set $Z$ is less than or equal to $n-3$.

To get the extent of the manifold $N$ which does not carry stable harmonic $S^{2}$, let us recall a famous index estimate result by Micallef and Moore 12 .

Proposition 2.6. Let $N$ be an $m$-dimensional Riemannian manifold with positive curvature on totally isotropic two-planes, Then any nonconstant conformal harmonic map $u: S^{2} \rightarrow N$ has index at least $\left(\frac{m}{2}\right)-\left(\frac{3}{2}\right)$, ind $(u) \geq\left(\frac{m}{2}\right)-\left(\frac{3}{2}\right)$. Furthermore, if $N$ had positive curvature operators or $(2,2)$-positive curvature operators instead of positive curvature on totally isotropic two-planes, then ind $(u) \geq m-2$.

Here ind $(u)$ denotes the Morse index of the harmonic map $u$. For the conditions: (1): positive curvature operators, $(2)$ : $(2,2)$-positive curvature operators and (3): positive curvature on totally isotropic two-planes, we refer to the paper [12].

Hence combining the above proposition with Theorem 1.2, we have

Theorem 2.7. Let $u \in H^{1,2}(\Omega, N)$ be a stable-stationary harmonic map, $\operatorname{dim} N=$ $m$. If the target manifold $N$ satisfies one of the following:

a) $N$ has positive curvature operators or $(2,2)$-positive curvature operators, $m>$ 2 ,

b) $N$ has positive curvature on totally isotropic two-planes, $m>3$, then the Hausdorff dimension of the singular set of $u$ is less than or equal to $n-3$.

For the case of a stable-stationary harmonic map, there are many contributions to Liouville-type theorems along these lines, for example, the condition 2superstrongly unstable manifold introduced by Wei and Yau 17]. At least the above theorem gives other types of results in this direction.

It is an interesting problem to reveal the relation of the regularity of the harmonic map with the curvature condition of the target manifold $N$ when $N$ has positive sectional curvature. Here our theorems gives evidence of these connections.

\section{ACKNOWLEDGEMENT}

The author would like to thank Professor Chang You Wang for valuable comments and suggestions.

\section{REFERENCES}

[1] W. Allard, An Integrality Theorem and a Regularity Theorem for surfaces whose First Variation with respect to a Parametric Elliptic Integrand is Controlled. Proceeding of Symp. In Pure Math. Vol. 44 (1986), AMS. 1-28. MR0840267 (87j:49077) 
[2] F. Bethuel, On the singular set of stationary harmonic maps. Manu. Math. 78 (1993) 417-443. MR.1208652 (94a:58047)

[3] L. C. Evans, Partial regularity for stationary harmonic maps into spheres. Arch. Rat. Meth. Anal. 116 (1990) 203-218. MR:1143435 (93m:58026)

[4] M. Giaquinta, E. Giusti, The singular set of the minima of certain quadratic functionals. Ann. Scuola Norm. Sup. Pisa CI. Sci. (4) 11(1984), no. 1, 45-55. MR0752579 (86a:49086)

[5] Deliang Hsu, Jiayu Li, On the regularity of stationary harmonic maps. Preprint.

[6] F. Hélein, Sur la régularité des applications faiblement harmoniques entre une surface et une variété riemannienne, C. R. Acad. Sci. Paris Sér. I. Math. 312 (1991) 591-596. MR1101039 (92e:58055)

[7] Min-Chun Hong, On the Hausdorff dimension of the singular set of stable-stationary harmonic maps, Commun. in Partial Differ. Equa. 24 (1999) 1967-1985. MR.1720786 (2000i:58027)

[8] Min-Chun Hong, Chang-you Wang, On the singular set of stable-stationary harmonic maps, Calc. Var. 9 (1999) 141-156. MR1714121 (2000i:58026)

[9] Jiayu Li, Gang Tian, A Blow-Up Formula for Stationary Harmonic maps, International Mathematics Research Notices, No.14 (1998) 735-755. MR1637101 (99d:58049)

[10] Fanghua Lin, Gradient estimates and blow-up analysis for stationary harmonic maps, Ann. of Math.(2) 149 (1999), No 3, 785-829. MR.1709303 (2000j:58028)

[11] R. Hardt, On the Singularity of Harmonic maps. Bulletin AMS 34 (1997) 15-34. MR 1397098 (98b:58046)

[12] M. J. Micallef, J. D. Moore, Minimal two-spheres and the topology of manifolds with positive curvature on totally isotropic two-planes, Annals of Math. 127 (1988) 199-227. MR 0924677 (89e:53088)

[13] T. Rivière, Everywhere discontinuous harmonic maps into spheres. Acta Math. 175 (1995), no. 3, 197-226. MR.1368247|(96k:58059)

[14] J. Sacks, K. Uhlenbeck, The existence of minimal immersions of 2-spheres. Ann. of Math. (2) 113 (1981), no.1, 1-24. MR0604040 (82f:58035)

[15] R. Schoen, K. Uhlenbeck, A regularity theory for harmonic maps. J. Diff. Geom. 17 (1982) 307-335. MF 0664498 (84b:58037a)

[16] S. W. Wei, Liouville theorem for stable harmonic maps into either strongly unstable, or $\delta$-pinched manifolds, Proceeding of Symp. In Pure Math. Vol. 44 (1986), AMS. 406-412. MR 0840289 (87i:58044)

[17] S. W. Wei, C. M. Yau, Regularity of $p$-energy minimizing maps and $p$-superstrongly unstable indices, J. Geom. Anal. 4 no. 2 (1994) 247-272. MF.1277509 (95f:58028)

Department of Mathematics, Shanghai Jiaotong University, Shanghai 200240, PeoPLE's Republic of ChinA

E-mail address: dlxu@sjtu.edu.cn 